\title{
RELIGION UND STAAT IN ISRAEL
}

\author{
Von ZE'Ev W. FALK
}

\section{Gesetzgebung}

Bei der Gründung des Staates Israel vor einem Vierteljahrhundert war das Verhältnis von Staat und Religion in der Verfassung der Mandatsregierung, dem Palestine Order-in-Council, 1922, geregelt. Die Bevölkerung des Landes war darin in Religionsgemeinschaften eingeteilt, von denen jede die schon aus türkischer Zeit bestehende Autonomie von neuem bestätigt erhielt. Artikel 17 dieser Verfassung verbot jede Diskriminierung der Religionen durch das Gesetz, und Artikel 83 sprach allen Menschen volle Gewissens- und Religionsfreiheit zu, eingeschränkt nur durch die zur Erhaltung der öffentlichen Ordnung und Sitten notwendigen Schritte.

Im Beschluß der Generalversammlung der Vereinten Nationen vom 29. November 1947 wurde die Anerkennung der in Palästina zu gründenden Staaten davon abhängig gemacht, daß diese die Gewissens- und Religionsfreiheit sowie Gleichberechtigung der verschiedenen Religionen zu wahren versprachen. Demgemäß enthält die Unabhängigkeitserklärung des Staates Israel vom 14. Mai 1948 die Proklamation der sozialen und politischen Gleichberechtigung der verschiedenen Bekenntnisse, Religions- und Gewissensfreiheit und Schutz aller heiligen Stätten.

Gleichzeitig erklärte sich Israel allerdings in diesem Dokument als der „jüdische“ Staat und nahm dabei auf den Beschluß der Vereinten Nationen, daß in Palästina ein jüdischer und ein arabischer Staat entstehen sollte, Bezug. Die Erklärung erwähnte die historisch-traditionelle Verbindung des jüdischen Volkes mit dem Lande, in dem seine geistige, religiöse und politische Form geprägt wurde, und schloß mit dem doppelsinnigen Hinweis auf den „Fels Israels“ (vgl. II. Sam. XXIII 3), der für religiöse und säkularisierte Juden gleich akzeptabel war.

Der Charakter des Staates als Heimstätte des jüdischen Volkstums und seiner Religion drückt sich im Rückkehrgesetz, 1950, aus, das jedem Juden das Recht zur Einwanderung und auf die Staatsangehörigkeit zuspricht. In einer Novelle von 1970 wird klargestellt, daß als Jude die von jüdischer Mutter geborene oder in religiöser Form zum Judentum übergetretene Person gilt, daß jedoch auch die nichtjüdischen Ehegatten, Kinder und Enkel zur Einwanderung und Staatsangehörigkeit berechtigt sind.

Damit hat der Gesetzgeber das Kriterium des Religionsgesetzes für die Zugehörigkeit zum Volkstum und zur Staatsangehörigkeit gewählt. Das Judentum wird als Einheit von Religion und Volkstum verstanden, und die Einwanderung, die Staatsangehörigkeit und das Personenstandsregister werden durch die vom Staat rezipierten Religionsgesetze geregelt.

Das Primat der jüdischen Religion drückt sich auch in den Gesetzen über die offiziellen Feiertage, das Verbot der Schweinezucht und die rituelle Verpflegung in der Armee aus. Jedoch ist in jedem dieser Gesetze für die Interessen der nichtjüdischen Bevölkerung gesorgt. Diese ist berechtigt, statt Sabbat und jüdischer Feiertage Freitag oder Sonntag bzw. die eigenen religiösen Feiertage zu halten. Das Verbot der Schweinezucht bezieht sich nur auf Teile des Landes mit überwiegend jüdischer Einwohnerschaft, wie auch die rituelle Verpflegung nur für jüdische Soldaten vorgesehen ist. Für diese gelten übrigens auch die Verordnungen des Generalstabs 
über die feldrabbinische Belehrung vor den hohen Feiertagen, die der Stärkung des religiösen Bewußtseins dient.

\section{Nichtjüdische Religionsgemeinschaften}

Obwohl das Judentum eine staatsreligionsmäßige Stellung einnimmt, sind auch den anderen Religionsgemeinschaften gleiche Chancen gegeben. Die größeren Religionsgemeinschaften werden paritätisch behandelt und genießen öffentlich-rechtlichen Status mit Verwaltungsautonomie und eigener Gerichtsbarkeit. Für Nichtjuden besteht ein spezielles Lehrprogramm im Rahmen des staatlichen Schulwesens und jede Diskriminierung von Religionsangehörigen bei Arbeitsnachweis und in der Hochschulbildung ist verboten. In Familienrechtsangelegenheiten von israelischen Staatsangehörigen gelten die Religionsgesetze der Parteien, ebenso werden d:ese Gesetze in Fragen der Stiftungen und in religiösen Angelegenhei ien angervanc'.. Natürlich unterliegt die religiöse Gerichtsbarkeit und das religiöse Recht den Str tsgesetzen, und wo letztere nicht berücksichtigt worden sind, kann das obcrste Gericht die Zwangsvollstreckung des Urteils unterbinden, da die religiösen Urteile auf die Vollstreckung durch die bürgerlichen Gerichte angewiesen sind.

Die weitestgehende Zuständigkeit ist seit der türkischen Zeit den mohammedanischen Gerichten gegeben, während jüdische und christliche Gerichtshöfe nur bei Ehe, Scheidung und Stiftungen ihrer Mitglieder ausschließlich zuständig sind und in anderen familienrechtlichen Angelegenheiten, wie auch wenn nicht al e Parteien die israelische Staatsbürgerschaft besitzen, auf das Einverstündnis der Parteien angewiesen sind.

Abgesehen von der oben genannten Form der Vollstreckung haben die christlichen Gerichtshöfe unumschränkte Autonomie, da die christlichen Religionsgemeinschaften es vorgezogen haben, die Verwaltung und Gerichtsbarkeit nicht durch das Staatsgesetz regeln zu lassen. In der Entscheidung Jidai v. Vorsitzender des Vollstrekkungsamtes $^{1}$ wurde die Frage behandelt, inwiefern der griechisch-katholische Gerichtshof staatlich anerkannt sein könne, da er von dem im feindlichen Ausland lebenden Patriarchen ernannt worden war. Das oberste Gericht kam zum Ergebnis, daß genannter Gerichtshof dennoch staatlich anzuerkennen sei, und zwar, weil der Patriarch nur im physischen Sinne im Ausland, im geistigen Sinne dagegen in Israel lebe.

Die Gerichtsbarkeit der Juden, Mohammedaner (Muslime) und Druzen ist durch das Staatsgesetz geregelt, ihre Richter sind Staatsbeamte und unterliegen den Weisungen des Staatsgesetzes, soweit es die Anwendung der Religionsgesetze beschränkt hat. Religiöse Splittergruppen können sich einen privatrechtlichen Rahmen geben und genießen gesetzlichen Schutz ihrer Gottesdienste. Auf Wunsch erhalten auch ihre Geistlichen die Berechtigung, Eheschließungen vorzunehmen, und seit 1969 besteht für sie sowie überhaupt für Mischehen die Zivilscheidung. Ein Teil dieser Gruppen entfaltet eine beträchtliche Missionstätigkeit besonders unter Hilfsbedürftigen und Kindern. Eine Novelle zum Rechtsfähigkeits- und Vormundschaftsgesetz von 1965 und das Gesetz zur Aufsicht über die Heime, 1965, beschränken diese Tätigkeit, ohne sie zu verbieten.

$1 \mathrm{PD}$ ix 135. 


\section{Judentum}

Die Stellung des Judentums im Staate und die staatliche Lösung religiöser Probleme führte zur Schwächung der demokratischen Traditionen in der jüdischen Gemeinde und zur Bürokratisierung ihrer Verwaltung. An Stelle der Ortsgemeinden traten seit Staatsgründung "religiöse Räte“, die zu 45 Prozent vom Religionsminister, zu 45 Prozent von der bürgerlichen Gemeinde und zu 10 Prozent vom Ortsrabbinat ernannt werden und deren Budget zu gleichen Teilen vom Ministerium und der bürgerlichen Gemeinde gedeckt wird. Nach einer Abmachung der Koalitionsparteien erhalten die religiösen Parteien 51 Prozent der Sitze, und die Repräsentanten der anderen Parteien haben ebenfalls ihre positive Stellung zum Judentum zu erklären. Obwohl diese Abmachung vom obersten Gericht ${ }^{2}$ für ungültig erklärt wurde, hält sich der Minister soweit wie möglich an sie $^{3}$.

Die Ortsrabbiner werden aus einer vom Oberrabbinat bestätigten Liste von einer Kommission ernannt, die sich aus Vertretern der bürgerlichen Gemeinde, des religiösen Rats und der Hauptsynagogen zusammensetzt. Das Oberrabbinat wird von einer aus 80 Rabbinern und 70 Laien bestehenden Kommission gewählt, die ihrerseits unter dem Einfluß der Parteien steht. Die gewählten Mitglieder des Oberrabbinats fungieren teilweise als Richter der rabbinischen Gerichte, teilweise als Ortsrabbiner. An ihrer Spitze stehen ein orientalischer und ein westlicher Oberrabbiner. Die Kompetenzen des Oberrabbinats sind teilweise durch das Gesetz und teilweise durch den Brauch festgelegt und schließen religionsgesetzliche Fragen, soweit sie nicht familienrechtlicher Natur und den rabbinischen Gerichten vorbehalten sind, Aufsicht über rituelle Schlachtung und Verpflegung, Prüfung von Rabbinatskandidaten und rabbinischen Richtern und Repräsentation ein.

Leider ist diese Körperschaft seit langer Zeit inaktiv. Sie leidet unter inneren Streitigkeiten und hat keine eigenen Beamten und kein Budget. So ist sie weitgehend vom Religionsminister und seiner Partei abhängig.

Dennoch konnte die National-Religiöse Partei, der der Minister angehört, nicht die Sympathien der Rabbiner gewinnen, und diese stimmen mehr mit der extremorthodoxen Agudat Israel Partei als mit der gemäßigten National-Religiösen Partei überein. Anfang Mai 1972 beschloß das Oberrabbinat, dem Minister nicht die Teilnahme an den Sitzungen zu gestatten und dadurch seinen Einfluß auf das Rabbinat zu reduzieren.

Die orthodoxen Rabbiner fühlen sich nämlich in dauernder Defensive gegenüber den Forderungen der areligiösen Öffentlichkeit und sehen sich zur immerwährenden Apologetik des Religionsgesetzes verpflichtet. Die Furcht vor der Reform des Religionsgesetzes spielt ebenfalls eine entscheidende Rolle und trägt zur aggressiven Stellung dem Staat gegenüber bei, von der noch die Rede sein wird. Das Oberrabbinat verdankt ja seine Position mehr dem staatlichen Gesetzgeber als eigener Tradition und ist deshalb besonders für den Vorwurf empfindlich, daß es in einer bestimmten Frage als Sprachrohr des Staates und nicht als würdiger Vertreter des spirituellen Judentums handle.

Wie gesagt, gibt es neben dem Rabbinat keine demokratische Vertretung der religiösen Gemeinde. Es existieren lokale Synagogenvereine und sogar ein Landesverband der Synagogen, aber beide haben keinerlei Einfluß auf die religiösen Angelegenheiten. So bleiben als Sprecher der religiösen gegenüber der politischen Macht nur die Rabbiner ohne organisierte Gefolgschaft oder die religiösen Parteien. Dadurch werden die religiösen Forderungen vom Publikum und von der Regierung 
oft nur als Kapricen oder politische Intrigen angesehen, auch wenn sie tatsächlich nicht nur als Mittel im opportunistischen Kampf um die Macht dienen sollen.

Ein anderer Grund für das negative Bild, das die religiösen Forderungen in der öffentlichen Meinung haben, ist das weitgehende Interessentum, das die Stellung religiöser Sprecher zu politischen Fragen bestimmt. Ein großer Teil der Studenten und Absolventen der rabbinischen Schulen genießt unbefristeten Aufschub der aktiven Militärdienstpflicht, und ein großer Teil der Mädchen ist aus religiösen oder gewissensmäßigen Gründen von der Dienstpflicht befreit. Die säkularistischen Kreise sehen darin ein Zugeständnis, das sie dem religiösen Sektor machen, wodurch dieser sich das moralische Recht verwirkt habe, die Allgemeinheit in seinem Sinne zu beeinflussen. Ahnlich sehen viele die religiöse Eheschließung und Gerichtsbarkeit als ein funktionales Zugeständnis an die Rabbiner an und leiten aus ihm die Forderung der Säkularisierung auf allen anderen Gebieten ab.

\section{Nichtorthodoxe Minoritäten}

Problematisch ist die Stellung der jüdischen religiösen Minoritäten und der konsequenten Atheisten. Die offiziellen Rabbiner und rabbinischen Richter gehören der orthodoxen Richtung an und bestehen auf dem staatlichen Monopol als alleinige Vertreter der jüdischen Religion. Dadurch sind auch die nichtorthodoxen Minoritäten der orthodoxen Gesetzesauslegung und -anwendung unterstellt.

1971 veröffentlichte die Regierung jedoch mit Einverständnis des Oberrabbinats einen Gesetzesvorschlag, der den 8000 Karäern, die seit dem neunten Jahrhundert die rabbinischen Gesetze bestreiten, im Rahmen der jüdischen Religionsgemeinschaft die Errichtung eigener Gerichtshöfe ermöglichen soll. Der Vorschlag hat bisher noch nicht Rechtskraft erlangt; viele Politiker fürchten die Folgen der gesetzlichen Trennung eines Teiles des jüdischen Volkes und wollen eine eventuelle Wiedervereinigung nicht ausschließen. Ein solches Gesetz wäre gewiß auch ein Präzedenzfall für die staatliche Anerkennung von anderen nichtorthodoxen Minoritäten.

Neben den Karäern gibt es nämlich die seit dem fünften vorchristlichen Jahrhundert neben dem Judentum bestehende Sekte der Samaritaner, die einige hundert Seelen zählt, und eine Reihe von konservativen und Reform-Gemeinden, die bisher nicht anerkannt sind. Ihre Existenzberechtigung hat sich erst letztens in der öffentlichen Meinung durchgesetzt. Seinerzeit wandten die religiösen Parteien alle Mittel an, um die Baugenehmigung einer Reformsynagoge in Jerusalem zu hintertreiben; nur die Drohung mit einem Antrag an das oberste Gericht führte schließlich zur Ausstellung der Baugenehmigung. Eine Stadtverwaltung wurde durch das oberste Gericht dazu gezwungen, der örtlichen Reformgemeinde ihren Saal zu vermieten ${ }^{4}$. Inzwischen genießen diese Gemeinden wenigstens privatrechtliche Gleichberechtigung und gewisse staatliche Zuwendungen.

Jüdische Atheisten versuchen die Öffentlichkeit durch private Eheschließungen, Anträge auf deren Anerkennung durch das oberste Gericht, demonstrative Sabbatentweihung und ähnliche Schritte auf die Notwendigkeit der Trennung von Religion und Staat und der Zivilehe aufmerksam zu machen. In Rogosinski v. Staat ${ }^{5}$

2 In BGZ 191/64, Albas v. Religionsminister, PD xviii (4) 603.

3 Vgl. BGZ 313/67, Axelrod v. Religionsminister, PD xxii (1) 80 .

4 BGZ 262/62, Perez v. Gemeinde Kefar Schemaryahu, PD xvi, 2101.

5 IE 450/70. PD xxvi (1) 129. 
zum Beispiel bestritten die Antragsteller die Rechtmäßigkeit der rabbinischen Gerichtsbarkeit und Eheschließung für nichtgläubige Juden aufgrund der Gewissensfreiheit. Das oberste Gericht wies jedoch den Antrag aufgrund des klaren Wortlautes des Gesetzes ab und verwies die Antragsteller an das Parlament. In ähnlicher Form versuchte der Antragsteller in Tamarin v. Staat ${ }^{6}$ als Israeli statt als Jude registriert zu werden. Auch dieser Antrag wurde abgewiesen, da es zur ethnischnationalen Definition eines einzelnen nicht genüge, daß er sich persönlich in einer bestimmten Weise definiere, sondern er ein Teil einer Gruppe sein müßte, die ihre Identität in dieser Besonderheit sähe. Doch selbst wenn sich eine solche Gruppe fände, die sich wie der Antragsteller als Israelis und nicht als Juden ansähen, so ist ihre Ablehnung der religiösen Elemente des Judentums nicht Grund genug, sich nicht als Juden zu deklarieren, denn der Gesetzgeber hat diese Möglichkeit nicht vorgesehen. Endlich solle das Prinzip der nationalen Selbstbestimmung nicht zur Zersplitterung benutzt werden.

Auch von christlicher Seite wird immer wieder betont, daß man jüdischer Nationalität sein und gleichzeitig der christlichen Religion angehören könne. Dieses Argument wurde vom obersten Gericht in Rufeisen v. Innenminister ${ }^{7}$ durch Mehrheitsbeschluß abgelehnt, weil es dem Volksempfinden widerspreche. Inzwischen bestimmt die oben genannte Novelle zum Rückkehrgesetz von 1970, daß nicht als Jude gelte, wer einer anderen Religion angehöre. Das Problem scheint jedoch noch immer nicht gelöst; so gibt es zum Beispiel momentan eine Gruppe von amerikanischen Negern, die sich als die wahren Nachkommen des biblischen Israels ansehen und daraufhin die Rückkehrerrechte für sich in Anspruch nehmen. Nach der heutigen Fassung des Gesetzes sind sie wohl, weil nicht von jüdischer Abstammung und nicht übergetreten, auszuschließen, doch ist die Form des Ubertritts nicht genau festgelegt. Praktisch erkennt der Staat auch die nicht der orthodoxen Form entsprechenden Úbertritte, die durch ausländische Reformrabbiner vorgenommen wurden, an, während für das Inland nur die orthodoxe Form gilt. Im Juli 1972 liegen zwei Gesetzesvorschläge der Oppositionsparteien von rechts und von links vor, die eine für den ausschließlich orthodoxen Ritus für den Übertritt und die andere für Einführung der Zivilehe in Fällen, wo ein religiöses Impediment besteht. Für jeden dieser Vorschläge besteht Sympathie bei einem Teil der Koalition, und die Regierung hat Schwierigkeiten bei der Bewahrung der parlamentarischen Disziplin.

\section{Staatliche Interventionen}

Obwohl das Religionsgesetz bereits vor Staatsgründung bestanden hat und auch außerhalb der Staatsgrenzen Anwendung findet, obwohl es also eine vom Staat unabhängige Stellung einnimmt, zieht der Staat auch religionsgesetzliche Fragen in seinen Kompetenzbereich. Im Rahmen des Erziehungsministeriums besteht eine fast autonome Abteilung für die religiösen Schulen innerhalb des staatlichen Grundschulsystems. In Fragen der religiösen Erziehung gesteht also die Regierung dem religiösen Sektor, der hier mit der Religiös-Nationalen Partei identisch ist, das Recht der Selbstverwaltung zu. Da aber die Zuteilung von Gebäuden und Inventar durch die allgemeine Verwaltung vorgenommen wird und die religiösen Schulen

6 IE 630/70. PD xxvi (1) 197.

7 BGZ 72/62. PD xvi 2428. 
mit der Religiös-Nationalen Partei identifiziert werden, so gibt es oft Klagen wegen ihrer Benachteiligung. Um staatliche Interventionen in dem religiösen Schulunterricht auszuschließen, gründete die rechts-orthodoxe Agudat Israel Partei ein eigenes „Unabhängiges Schulsystem“. Inzwischen wird dieses System ebenfalls zu 90 Prozent vom Staat subventioniert und untersteht dadurch der Aufsicht des Erziehungsministeriums.

Religiöse Kreise sehen das Rabbinerwahlsystem, das den Regierungsparteien den Ausschlag gibt, als Eingriff in die religiöse Sphäre an. Als ein unberechtigter Verstoß gegen das Religionsgesetz gilt die Arbeitsdienstpflicht der Mädchen. Es besteht allerdings die Möglichkeit der Befreiung aus Gewissensgründen, wenn eine Kommission vom guten Glauben der Antragstellerin überzeugt ist. Jüngst erklärte eine Gruppe von Mädchen, die die Befreiung nicht erhalten hatten, daß sie das vom Oberrabbinat veröffentliche Verbot des Mädchenarbeitsdienstes nicht übertreten wollten und es vorzogen, ins Gefängnis zu gehen. Große Verbitterung ruft das Gesetz über die Anatomie und Pathologie hervor, das gestattet, die Sektion gegen den Willen des Verstorbenen und der Hinterbliebenen vorzunehmen, wenn drei Ärzte es für notwendig halten. 1966 verbot das Oberrabbinat zusammen mit einer Anzahl Rabbinern jede Sektion ohne spezielle rabbinische Erlaubnis und erklärte die gesetzliche Regelung als ein Unrecht, gegen das man sich auflehnen muß.

Das Gesetz über die Ruhetage gestattet dem Arbeitsminister, die Sabbatruhe in gewissen Betrieben aufzuheben und dadurch religiösen Menschen solche Arbeitsplätze zu verschließen. In Meron v. Arbeitsminister ${ }^{8}$ versuchte der Antragsteller, die Sabbatruhe auf die Television anzuwenden und den Dispens des Ministers für ungültig erklären zu lassen. Der Antrag wurde jedoch mangels persönlichen Interesses des Antragstellers abgewiesen.

In seiner Eigenschaft als Überprüfungsinstanz der religiösen Gerichte interveniert das oberste Gericht nicht selten in rein religiösen Angelegenheiten. Zwar hat es eigentlich nur die Kompetenz des religiösen Gerichts und nicht die materiell-rechtliche Seite des Urteils zu prüfen (Paragraph 7 [b] [4] des Gesetzes über die Gerichte, 1957), doch betätigt es sich oft faktisch als Berufungsinstanz und unterzieht die rabbinische Quelleninterpretation seiner Kritik. Diese Tendenz besteht besonders bei den nichtreligiösen Mitgliedern des obersten Gerichts, die dadurch ihr Mißfallen über den religiösen $\mathrm{Z}$ wang zum Ausdruck bringen.

So erklärte das oberste Gericht eine Entscheidung des Oberrabbinats, daß eine Schlachterei rituell unzulässig sei, für ungültig9. In diesem Falle weigerte sich das Oberrabbinat, vor dem obersten Gericht zu erscheinen, da letzteres in rituellen Fragen nicht kompetent sei. Das oberste Gericht setzte die Verhandlung dennoch fort, weil das Oberrabbinat eine öffentliche Funktion ausübe und deshalb wie jede andere solche Institution gemäß Paragraph $7(b)(2)$ des Gesetzes über die Gerichte, 1957 der Prüfung durch das oberste Gericht unterstehe. Der Antragsteller einigte sich jedoch mit dem Oberrabbinat und nahm den Antrag zurück.

In einem anderen Falle, Streit v. Oberrabbiner ${ }^{10}$, verbot das oberste Gericht dem Oberrabbiner, einen Bigamiedispens zu erteilen, obwohl dieser nach jüdischem Recht möglich war. Das oberste Gericht sah in diesem Akt eine administrative Entscheidung aufgrund des Strafrechtsreform(Bigamie)-Gesetzes, 1959, da nach rabbinischer Tradition ein Dispens von 100 Rabbinern gegeben werden müßte. Obwohl das oberste Gericht den Akt als religionsgesetzlich ansah, forderte es den Ober- 
rabbiner auf, gemäß dem bürgerlichen Gesetz zu handeln. In einer späteren Entscheidung ${ }^{11}$ erklärte die Mehrheit des obersten Gerichts, daß das bürgerliche Gesetz dem Oberrabbiner die freie Anwendung des rabbinischen Rechts gestattet habe und er deshalb den Dispens erteilen dürfe.

Die Mehrheit des obersten Gerichts beschloß in Rodnitzki v. Rabbinisches Obergericht ${ }^{12}$, eine privatim geschlossene Ehe zwischen einem Manne, dem wegen priesterlicher Abstammung die Ehe mit der geschiedenen Frau verweigert worden war, gegen die Ansicht des rabbinischen Gerichts als gültig zu erklären. Dabei lehnte das oberste Gericht die Begründung des rabbinischen Gerichts ab, nämlich daß die bei der Eheschließung fungierenden Zeugen als Sabbatübertreter religionsgesetzlich inkompetent seien, und erklärte diese Ansicht als mit der Meinung der Bevölkerungsmehrheit unvereinbar. Das Gesetz schreibe zwar für Juden die religiöse Eheschließungsform vor und verbiete also private Eheschließungen, jedoch ließe sich dadurch nicht das rein religiöse Eheverbot aufzwingen. Wenn also ein religiöses impedimentum impediens besteht und die betreffende Person die Ehe privat geschlossen hat, so mußte das rabbinische Gericht nachträglich die Ehe anerkennen und wenn es sich weigerte, so besteht ein Grund für die Intervention des obersten Gerichts. Allerdings wendet sich das Urteil nur an den Minister, dem das Personenstandsregister untersteht, und nicht an das rabbinische Gericht selbst.

\section{Rabbinische Interventionen}

Andererseits handelt auch das Rabbinat und die Rabbinische Gerichtsbarkeit oft als wenn sie nicht dem Staatsgesetz unterstehen würden. Tatsächlich besteht von jeher das Problem der doppelten Loyalität der rabbinischen Richter; es drückt sich unter anderem im Gesetz über die rabbinischen Richter aus. Während in Paragraphen 11 und 13 des Richtergesetzes, 1953 gesagt ist, daß der Richter zu versprechen habe, dem Staate und seinen Gesetzen treu zu sein, und daß er keiner Autorität als der des Gesetzes unterliege, so heißt es in Paragraphen 10 und 12 des Gesetzes über die Rabbinischen Richter, 1955, daß der Rabbinische Richter verspreche, dem Staate treu zu sein, und daß er keiner Autorität als der des Gesetzes, nach dem er richte - also der des Religionsgesetzes - , unterliege.

Obwohl das Frauengleichberechtigungsgesetz, 1951, ausdrücklich die religiösen Gerichte verpflichtet, nehmen die rabbinischen Richter nicht davon Kenntnis und berufen sich ausschließlich auf die rabbinischen Rechtsquellen ${ }^{13}$. Ahnlich handelt übrigens auch das mohammedanische Gericht ${ }^{14}$.

Das Gesetz über das Ehemindestalter, 1950, verbietet die Zuwiderhandlung, ohne die Gültigkeit der einmal geschlossenen Ehe zu annullieren. Es bestimmt deshalb, daß dieser Umstand auf Antrag eines Elternteils, des Vormunds oder des Fürsorgebeamten vom Gericht gemäß dem Personenstandsrecht der Parteien als Scheidungsgrund anzunehmen sei. Bisher ist jedoch nie ein solcher Antrag gestellt worden. Bei jüdischen Eheleuten ist das rabbinische Gericht zuständig, und dieses räumt den genannten Personen keine Berechtigung ein, die Scheidung zu beantragen.

10 BGZ 301/63. PD xviii 598.

11 DN 10/69, Boronowski v. Oberrabbiner, PD xxv (1) 7.

12 BGZ 51/69. PD xxiv (1) 704

13 Vgl. DN 23/69, Josef v. Josef, PD xxiv (1) 792, 809.

14 BGZ 187/54, Berya v. Kadi, PD ix 1193. 
Als 1964 die aus Indien eingewanderten „Bene Israel“ vom Rabbinat nur aufgrund individueller Untersuchung als Juden zur Eheschließung zugelassen wurden, veranstalteten sie einen Proteststreik, woraufhin das Parlament während der Ferien zusammentrat und das Rabbinat um eine liberalere Stellungnahme bat. Darauf antwortete der Oberrabbiner mit der Drohung, daß er bei weiterer Intervention seinerseits auch diese beschränkte Zulassung der "Bene Israel“ aufheben würde, so wie es die rechts-extremen Rabbiner längst forderten. Daraufhin verzichtete das Parlament auf weitere Aktionen in der Angelegenheit.

Wie schon erwähnt, veröffentlichte das Oberrabbinat 1966 ein Verbot jeder anatomischen und pathologischen Sektion ohne vorherige spezielle rabbinische Erlaubnis und trat damit gegen das Anatomie- und Pathologie-Gesetz, 1953, auf, das seinerzeit mit Einverständnis des vorigen Oberrabbiners angenommen worden war.

1971 weigerte sich ein rabbinisches Gericht, zwei Schwestern die Ehefähigkeit zuzuerkennen, nachdem es sich herausgestellt hatte, daß ihre Mutter bei der Empfängnis nicht mit dem Vater, sondern mit einem anderen Manne verheiratet war. Auf die Intervention des Sicherheitsministers, an den sich die Geschwister während ihrer Militärdienstzeit gewandt hatten, versprach der Oberrabbiner, die Angelegenheit erneut zu prüfen. Es gelang ihm jedoch nicht, unter den Rabbinern einen Gerichtshof zu finden, der das erste Urteil aufheben würde. Gerade weil die öffentliche Meinung eine erleichternde Stellungnahme fordert, reagieren die meisten Rabbiner in der entgegengesetzten Richtung. Einerseits wollen sie sich nicht von der traditionellen Interpretation des Religionsgesetzes abbringen lassen und andererseits fürchten sie mehr die Kritik ihrer Kollegen von rechts als die des Publikums von links.

Ende 1971 versammelte sich eine Anzahl von Rabbinern, Rabbinischen Richtern und anderen Personen, die der rechts-orthodoxen Agudat Israel Partei nahestehen, und wählten auf eigene Faust ein Mitglied des Rabbinischen Obergerichts als "Oberrabbiner Jerusalems". Sie begründeten diese eigenmächtige Verletzung der Wahlprozedur damit, daß es religionsgesetzlich verboten sei, bei der Wahl des Rabbiners Laien zu beteiligen, besonders Vertreter nichtreligiöser Parteien. Es ist bezeichnend, daß sich rabbinische Richter an diesem Akt beteiligen und wählen lassen konnten, ohne daß sie wegen Vergehens gegen das Staatsgesetz zur Ordnung gerufen wurden. Inzwischen begann der „Religiöse Rat“ mit der Vorbereitung der offiziellen Rabbinerwahl, aber es ist fraglich, ob es ihm gelingen wird, das fait accompli zu überwinden.

Nachdem das Oberrabbinat seit Jahren zum Widerstand gegen das Frauenarbeitsdienstgesetz und erst recht gegen den Militärdienst von Mädchen aufgerufen hatte, versuchte die Regierung 1971 den Arbeitsdienst in freiwilliger Form durchzuführen. Daraufhin wurde beim rabbinischen Gericht gegen den religiös-nationalen Minister ein Klage mit dem Antrage eingereicht, daß ihm die Beteiligung an den Regierungssitzungen über den Frauenarbeitsdienst verboten werden solle, weil dieser dem Religionsgesetz widerspreche.

Obwohl das Staatsgesetz die rabbinischen Gerichte nur in familienrechtlichen und nicht in politischen, staatsrechtlichen Fragen ermächtigt, sandte das Gericht dem Minister die Vorladung, und dieser versprach, dem Termin Folge zu leisten. Nun wandte sich der Staatsanwalt an das Oberste Gericht und erhielt eine einstweilige Verfügung gegen das rabbinische Gericht wegen dessen Unzuständigkeit. Daraufhin schloß das rabbinische Gericht die Akte, doch der Kläger legte beim rabbinischen 
Obergericht Berufung ein. Letzteres entschied aufgrund der Zuständigkeit des Religionsgesetzes, die es ermöglicht, selbst einen König oder Hohen Priester vor das Gericht zu zitieren, und gab dem rabbinischen Gericht der ersten Instanz auf, die Klage zu hören. Darauf wandte sich der Staatsanwalt abermals an das Oberste Gericht, erlangte wieder eine einstweilige Verfügung, und dann nahm der Kläger seine Klage zurück.

Die Handlungsweise des rabbinischen Gerichts wurde in der Presse vom früheren Präsidenten des Obersten Gerichts scharf gerügt. Er betonte, daß die rabbinische Gerichtsbarkeit nur aufgrund des Staatsgesetzes existiere, und daß sie nicht außerhalb ihres Kompetenzbereiches handeln dürfe. Private Rabbiner könnten den Minister kritisieren, doch nicht staatlich ernannte und besoldete rabbinische Richter. Das jüdische Religionsgesetz gelte nur soweit als es vom staatlichen Gesetzgeber rezipiert sei und könne deshalb nicht gegen eine staatsrechtliche Entscheidung angeführt werden ${ }^{15}$. Ähnliches hatte der Präsident bereits in Balaban v. Balaban ${ }^{16}$ behauptet, als er davon sprach, daß nichts den säkularen Gesetzgeber hindern könne, eine Norm des Religionsgesetzes zu ändern. Solange keine Verfassung existiere, meinte er, die so etwas verbietet, wäre der säkulare Gesetzgeber (das Parlament) allmächtig.

In ähnlichem Sinne schreibt der Jerusalemer Ordinarius der Philosophie ${ }^{17}$. Er gesteht zwar dem Religionsgesetz einen vom Staate unabhängigen Rahmen zu und betont, daß es nicht auf demokratischen Voraussetzungen beruht, doch sei die Handlung des rabbinischen Gerichts selbst vom Religionsgesetz her nicht zu rechtfertigen. Die rabbinische Gerichtsbarkeit sei keine göttliche, sondern eine menschliche Einrichtung, und als solche unterliege sie der Regel, daß nur der Staat Zwang ausüben dürfe und alle vom Staate abgeleiteten Institutionen nur im von ihm verliehenen Kompetenzrahmen $Z$ wang anwenden können.

Wie ich zu zeigen versucht habe ${ }^{18}$, werden beide Verfasser dem Problem von Religion und Staat nicht gerecht. Der staatliche Gesetzgeber kann das Religionsgesetz ebenso wenig wie fremdes Recht, Glaubenslehren, wissenschaftliche Thesen und ästhetische Lehren abändern. Er kann wohl den verwaltenden und Recht sprechenden Organen Anweisung geben, was sie von den genannten Disziplinen anzuwenden haben, aber damit wird "das Ding an sich" nicht berührt. Wenn sich das Rabbinat auf das Religionsgesetz gegen den Staat beruft, so setzt es damit die Traditionen der Propheten und Pharisäer fort, die sich nicht scheuten, den Machthabern Vorhaltungen zu machen.

Wo ein Widerspruch zwischen den religiösen und staatlichen Autoritäten entsteht, müßte ein religionstreuer Minister unter Umständen zurücktreten. Denn auch wenn er nicht dem Rabbinat gegenüber verantwortlich ist, so bedeutet dessen Entscheidung doch einen schwerwiegenden Grund gegen das Verbleiben in der Regierung.

So wie ein staatlich besoldeter Rabbiner es sich nicht nehmen lassen kann, seine Gemeinde über die Notwendigkeit der religiösen Erziehung zu belehren und vielleicht sogar vor den Wahlen zugunsten der religiösen Parteien zu sprechen, haben auch die rabbinischen Richter die Pflicht, ihre Meinung zu äußern. Allerdings muß klargestellt werden, daß sie bei solcher Beschäftigung mit außerhalb des staatlichen Kompetenzbereichs liegenden Fragen auf die ursprüngliche religiöse

15 Ma'ariv, Abendzeitung, 8. 11. 1971.

16 IE $313 / 59$. PD xiv 285.

17 Ma'ariv, 4. 2. 72, 6. 4. 72.

$18 \mathrm{Ma}$ ariv, 18. $10.71,6.3 .72$. 
Kompetenz zurückgreifen, die trotz der staatlichen Gesetzgebung nicht zu bestehen aufgehört hat. Würde der Staat ihnen daraufhin die staatliche Autorität nehmen, so bliebe noch immer ihre religiöse Autorität in den Augen der religiösen Gesellschaft. Der Staat könnte allerdings die Rabbiner vor die Wahl stellen, welche von beiden Autoritäten sie für sich in Anspruch nehmen wollen, aber wenn er hochstehende Persönlichkeiten in seinen Dienst nehmen will, so muß er ihnen auch Rede- und Gewissensfreiheit zugestehen.

Die Unabhängigkeit des Rabbinats kann eine der „checks and balances“ sein, die uns vor dem totalitären Staat bewahren sollen. Dazu kommt, daß die Geschichte des Volkes Israel und damit auch des Staates nur dann einen Sinn haben, wenn in gewisser Weise die Kontinuität der religiösen Aufgabe gewahrt bleibt. Wenn viele Juden ihrer Aufgabe als Gottesdiener nicht nachkommen, so haben durch die Verbundenheit des Staates mit der Religion auch sie Anteil an ihrer Mission. Die Trennung von Staat und Religion würde dem Judentum in Israel die Verbindung mit der Diaspora und der Geschichte rauben und die Zukunft des Staates und Volkes gefährden. In diesem Sinne erklärte Ministerpräsidentin Golda Meir Ende April 1972 vor der Zentrale der Arbeitspartei: „Der Staat Israel, der sich nicht für die Existenz des jüdischen Volkes verantwortlich fühlte, würde mir nichts bedeuten. Er hätte keine Existenzberechtigung, und es wäre schade um jeden Tropfen vergossenen Blutes. Durch die Einführung der Zivilehe würde die Zahl der Mischehen in der Diaspora furchtbar zunehmen."

Doch das Problem, das sich uns stellt, ist, wie sich die religiöse Tradition des Judentums im modernen, pluralistischen Staat verwirklichen läßt, ohne die individuelle Freiheit und das Gewissen derjenigen zu verletzen, die sich mit seiner Lehre nicht identifizieren, und wie „ein Königreich von Priestern und ein heiliges Volk" in einer säkularen Welt bestehen kann. Es bedarf dafür nicht so sehr der dem am Jenseits ausgerichteten Christentum enstprechenden Trennung von Staat und Religion als eines modus vivendi von gegenseitiger Kompromißbereitschaft.

Die gegenseitigen Beziehungen von Staat und Religion treten seit Oktober 1972 in ein neues Stadium, seitdem nämlich der ehemalige Armeerabbiner Schelomo Goren mit Hilfe der Arbeiterpartei zum Oberrabbiner gewählt wurde. Diese Wahl stand unter dem Vorzeichen seiner liberalen Interpretation des Religionsgesetzes, die den Interessen des säkularen Publikums entgegenkommt.

Obwohl diese Wahl von orthodoxer Seite als unberechtigte Intervention in religiöse Angelegenheiten angesehen wird, spiegelt sie nur das größere Interesse der Offentlichkeit an diesen wider. Von jeher wurden die Rabbiner nicht von einer Hierarchie ernannt, sondern von der Gemeinde gewählt. Dadurch hatte die Gemeinde einen gewissen Einfluß auf ihre geistige Führung und selbst auf die Interpretation des Religionsgesetzes durch diese Führung. Die Wahl ist ein Mißtrauensvotum der Mehrheit gegenüber der bestehenden rabbinischen Ordnung und versucht ohne Trennung von Staat und Religion durch eine Reinterpretation der religiösen Quellen zu einem Ausgleich zu gelangen.

Als Erstes wandte sich der neue Oberrabbiner an die für Trennung eintretenden Koalitionsparteien und bat sie um Geduld, so daß er Gelegenheit zur Entwicklung einer liberalen Haltung bekäme. Dann umging er durch Bildung eines Sondergerichtshofes die strenge Praxis des rabbinischen Gerichts und hob dessen unpopuläres Eheverbot in einem bestimmten Falle auf. Während die Regierung, die Mehrheit des Publikums und ein Teil der Rabbiner diesen Schritt begrüßen, sieht das orthodoxe Publikum in ihm einen Eingriff in die rabbinischen Kompetenzen und eine Uber- 
tretung des Religionsgesetzes. Ein Abgeordneter der orthodoxen Agudat Israel Partei kündigte bereits die Bildung von Separatgemeinden an und schloß sich damit der von religiöser Seite geforderten Trennung von Religion und Staat an.

Es bleibt abzuwarten, inwiefern es Rabbiner Goren gelingen wird, das Band zwischen den verschiedenen Meinungen auf der Basis der religiös-nationalen Einheit Israels aufrechtzuerhalten. 
Court in Probate, Divorce and Matrimonial Causes and proceedings shall be exercised in conformity with the law and practice for the time being in force in England. Changes in the law of England automatically affected Ghanaian divorce law.

Ordinance marriage differs from the traditional and Islamic law marriage in basic conceptions and effects. Whilst Ordinance marriage is strictly monogamous, the other forms of marriage are potentially polygamous. The grounds for divorce in English law are limited but traditional law seems to know of no such limitation.

Divorce in English law was based on the principle of fault and the party seeking to dissolve a marriage had to prove that the other party had been guilty of a matrimonial offence. The Matrimonial Causes Act of 1971 has changed the position by making proof of the breakdown of the marriage the sole ground for divorce. Henceforth, it will no longer be the task of the courts to pronounce one party guilty and the other innocent but to find out whether the marriage has broken down beyond all repair.

\section{Conflict between Customary and Non-Customary Systems of Law:}

\section{Preliminary Observations}

\section{By I. Oluwole Agbede}

This essay attempts to provide a better understanding of the statutory rules designed to resolve the conflict between customary and non-customary systems of laws in Nigeria. In particular it attempts to establish that under the statutory rules non-natives are amenable to customary law contrary to the view expressed in earlier cases. It also shows that the influence of customary law varies from one field of the law to another. For example, customary law is fully developed and still dominant in the fields of land transactions and family relations. It is rudimentary in the fields of tort and contract and practically silent in the fields of company law, banking, road traffic, bankruptcy, insurance, etc. and completely abolished in the field of criminal law and punishment.

The essay also shows that the choice of law rules only apply to the superior courts in which case the customary courts are not bound by them. Finally, it shows that the rules are, in the main, merely presumptions which may be rebutted in suitable cases. In short, the rules are not rigid or inflexible as the courts tend to make them.

The essay also highlights the lack of research work until lately in the area of internal conflict of laws in common law Africa generally in contrast to the intensive work done in this area by continental jurists.

\section{Religion and the State in Israel}

By ZE'EV W. FALK

The State of Israel attempts the combination of the ideas of a "Jewish state" with that of the freedoms of religion and conscience, parity of religious communities and protection of holy places. While non-Jewish communities enjoy religious 
autonomy, Jewish religious affairs are settled bureaucratically and there is little contact between public opinion and the rabbinate. A special difficulty results from Jewish non-orthodox minorities.

As a result of the existing set-up the state intervened in matters of religious education, national service of women, Sabbath, rabbinical jurisdiction and the election to rabbinical office. Rabbinical courts have disputed the legality of state laws dealing with woman's equality, age of marriage, anatomy, national service of women and impediments of marriage.

In spite of the difficulties in combining Jewish religion with liberal democracy, the author rejects separation of state and religion as being inadequate to the special character of Israel.

\section{The Integrative Role of the Supreme Court in Israel - some Relevant Cases}

\section{By Joseph Ben Menashe}

The judicature, particularly in countries where the judiciary system depends largely on precedent, can play an important role in the process of modernization. This paper examines the specific role played by the Supreme Court of Justice in Israel in this context with regard to two problems: civil and religious marriages and registration of nationality. Both problems are paradigmatic for the modernizing process and have given rise to intense political and religious controversy. Both questions have been affected by religiously orthodox, "clerical, racist and coercive" legislation forced upon cabinet and parliament by a powerful (i. e. indispensable for coalition building) minority. The author analyzes the most important recent cases on this subject.

\section{Constitutional Amendments in Turkey - A Reply}

By BASSAM TiBI

In 1924 the nationalist movement led by Atatürk succeeded in dissolving the Sultanate - Caliphate and in establishing the republic. Until 1950, when they had to cede to the party of the Turkish bourgeoisie, the Democratic Party (DP), power was held by the Kemalists, i. e. the Republican Peoples Party (CHP). The latter's policy of reform and the experiment in transforming society from above through decree, were ended. The DP broke up the state industrial projects, stopped attempts at secularization and suspended democratic freedoms. In 1960 the military intervened, displaced the DP and introduced constitutional freedoms. This liberalization led to the rise of a powerful opposition which fought for social change. Interposing again, the military this time did away with the freedoms guaranteed by the constitution through changing the latter. Their obvious intention was to render the growing opposition illegal. E. E. Hirsch interprets this constitutional change as the attempt to disarm the "enemies of freedom", by which term he means: any opposition. The present paper challenges this interpretation. It confronts the statements made by Hirsch with historical developments and proves the former to be untenable. It shows that the 1971 amendments to the Turkish constitution do not constitute a "protection of democracy" as claimed by Hirsch but, by revoking democratic fundamental rights, its dissolution. 